SLAC-PUB-7544

May 1997

CONF-970503--277

\title{
Waveguide Stub-Line Tuning of RF Cavities with Heavy Beam Loading *
}

\author{
P. Krejcik \\ Stanford Linear Accelerator Center, Stanford University, Stanford CA 94309
}

\begin{abstract}
A method is proposed for using adjustable, waveguide stub-line tuners to match the load impedance of the cavity plus beam to the power source, without the need to detune the cavity. Adjustable stub-line tuners are shown to be able to completely match the resistive and reactive parts of the load impedance to a generator under all conditions of beam loading, ensuring optimum power transfer between generator and cavity. This technique may have advantages in high-current storage rings such as the SLC damping rings and the new PEP II storage rings. The coupled-bunch instability driven by the fundamental mode of the cavity is re-appraised in this coupling scheme in which the cavity is no longer detuned. The consequences of this matching scheme are also considered for the beam loading stability limit.

DISCLAIMER
\end{abstract}

\begin{abstract}
This report was prepared as an account of work sponsored by an agency of the United States Government. Neither the United States Government nor any agency thereof, nor any of their empleyees, makes any warranty, express or implied, or assumes any legal liability or responsibility for the accuracy, completeness, or usefulness of any information, apparatus, product, or process disclosed, or represents that its use would not infringe privately owned rights. Reference herein to any specific commercial product, process, or service by trade name, trademark, manufacturer, or otherwise does not necessarily constitute or imply its endorsement, recommendation, or favoring by the United States Government or any agency thereof. The views and opinions of authors expressed herein do not necessarily state or reflect those of the United States Government or any agency thereof.
\end{abstract}

Contributed to the 1997 Particle Accelerator Conference, Vancouver, B.C., Canada, May 12-16, 1997.

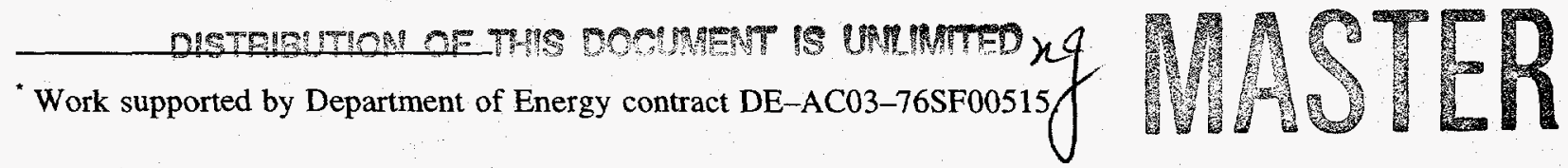




\title{
Waveguide Stub-Line Tuning of RF Cavities with Heavy Beam Loading*
}

\author{
P. Krejcik, \\ Stanford Linear Accelerator Center, Stanford University, Stanford, CA 94309 USA
}

\begin{abstract}
A method is proposed for using adjustable, waveguide stub-line tuners to match the load impedance of the cavity plus beam to the power source, without the need to detune the cavity. Adjustable stub-line tuners are shown to be able to completely match the resistive and reactive parts of the load impedance to a generator under all conditions of beam loading, ensuring optimum power transfer between generator and cavity. This technique may have advantages in high-current storage rings such as the SLC damping rings and the new PEP II storage rings. The coupled-bunch instability driven by the fundamental mode of the cavity is reappraised in this coupling scheme in which the cavity is no longer detuned. The consequences of this matching scheme are also considered for the beam loading stability limit.
\end{abstract}

\section{INTRODUCTION}

The beam in an RF cavity is seen by the generator (klystron) as a complex load impedance. The reactive component of this beam load impedance is determined by the synchronous phase angle, $\phi_{S}$, of the beam and consequently held fixed by other parameters of the accelerator. In high-current storage rings it is necessary to fully match this complex load to the generator to ensure efficient transfer of power.

In a typical storage ring cavity this is done in two steps by matching the real, or resistive, part with the coupling ratio at the cavity port and secondly detuning the cavity to match the imaginary, or real, part of the load. The resistive component of the match is not typically adjustable and is fixed by the geometry of the cavity coupler. In such cases the matching is only optimized for one design beam intensity. The reactive matching through cavity detuning can be achieved with a mechanical tuner and adjusted according to the average beam current.

The impedance seen by the generator determines the power transfer efficiency to the beam, but the impedance seen by the beam determines the stability of the beam. In high-current, multiple bunch "factory" machines the cost and feasibility of feedback systems to damp these instabilities must be weighed against the cost of the installed RF power. Coupled bunch instabilities are driven by the asymmetric impedance at the betatron sideband frequencies which is a direct consequence of detuning the cavity[1]. The beam loading stability threshold is also lowered as the cavity is detuned to obtain efficient matching. As the cavity impedance angle is increased with the beam intensity the generator component, $V_{G}$, of the cavity

\footnotetext{
- "Work supported by Department of Energy Contract DE-AC03-76SF00515.
}

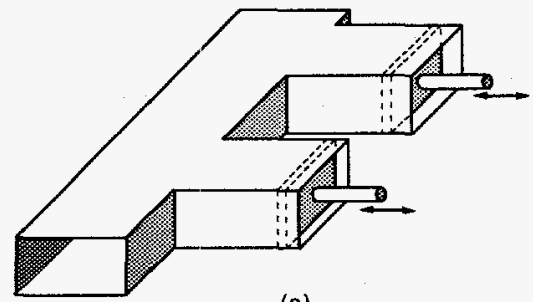

(a)

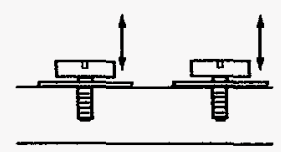

(b)
Figure 1: a. Adjustable stub lines perpendicular to the waveguide between the cavity and klystron, $b$. an equivalent alternative using adjustable posts in the waveguide.

voltage becomes increasingly out of phase with the total cavity voltage, $V_{C}$, until the Robinson limit is reached where $V_{G}$ is $180^{\circ}$ out of phase to the beam current, $I_{B}$, and no longitudinal focusing remains $[2,3]$.

The load matching discussed in this paper utilizes adjustable stub lines placed in the waveguide between the cavity and the klystron. Rather than adjusting the cavity parameters to match the generator, the stub lines add an extra degree of freedom to transform an unmatched load to its source. Once the new matching conditions have been established with the stub lines the impedance, seen by the beam, of the cavity plus the generator is analyzed and the conditions for beam stability described.

The stub line matching of the load impedance to its power source does appear to have the advantage of providing all of the adjustment outside of the cavity vacuum. Furthermore, the matching for both the resistive and reactive components can be adjusted for any beam intensity. In the conventional coupling scheme only the reactive matching is adjustable. The capability to vary the resistive component of the match becomes more critical for machines where the synchronous phase is large.

\section{THE BEAM AND CAVITY LOAD}

The equivalent circuit model of the cavity resonator coupled by a transmission line to a generator such as a klystron, shown in figure 2 , has been described in detail by Wilson [4]. In this model the fundamental mode of the cavity is represented by a parallel RLC circuit [5]. The beam current $I_{B}=2 I_{O} e^{-j\left(\alpha \boldsymbol{x}+\phi_{B}\right)}$, where $\phi_{B}=\frac{3 \pi}{2}-\phi_{S}$, can be represented as a load admittance, $Y_{B}$, with real and imaginary components

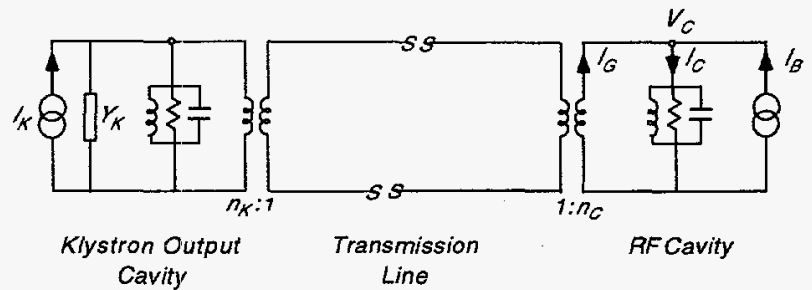

Figure 2: Equivalent circuit model of klystron, transmission line and cavity (after Wilson [4]). 


$$
\begin{aligned}
& \operatorname{Re}\left(Y_{B}\right)=\operatorname{Re}\left(\frac{I_{B}}{V_{C}}\right)=-\left|\frac{I_{B}}{V_{C}}\right| \sin \phi_{S} \\
& \operatorname{Im}\left(Y_{B}\right)=\operatorname{Im}\left(\frac{I_{B}}{V_{C}}\right)=\left|\frac{I_{B}}{V_{C}}\right| \cos \phi_{S}
\end{aligned}
$$

The total current in the cavity, $I_{C}$, is the vector sum of the generator and beam currents, $\hat{I}_{C}=\hat{I}_{G}+\hat{I}_{B}$. The combined impedance contributions from the beam and the cavity gives a total real and imaginary admittance of

$$
\begin{aligned}
& \operatorname{Re}\left(Y_{G}\right)=\operatorname{Re}\left(\frac{I_{G}}{V_{C}}\right)=\frac{1}{R_{s h}}\left(1+\left|\frac{I_{B}}{V_{C}}\right| R_{s h} \sin \phi_{S}\right) \\
& \operatorname{Im}\left(Y_{G}\right)=\operatorname{Im}\left(\frac{I_{G}}{V_{C}}\right)=-\frac{1}{R_{L}} \tan \phi_{Z}+\left|\frac{I_{B}}{V_{C}}\right| \cos \phi_{S}
\end{aligned}
$$

Matching of the load to the generator for optimum power transfer is done in two independent steps. To match the real part of the impedance requires finding $\beta_{\mathrm{C}}$ such that the admittance in equation (2), when transformed to the generator side of the transformer, becomes equal to the characteristic line admittance. This gives

$$
\beta_{C}=1+\left|\frac{I_{B}}{V_{C}}\right| R_{s h} \sin \phi_{S}
$$

In order to match the imaginary part of the impedance we find that the beam has introduced a reactive term that in present designs is compensated by reactive tuning of the cavity $[6,7]$. The cavity has a non-zero imaginary admittance when tuned away from its resonant frequency,

$$
Y_{c}(\omega) \approx \frac{1}{R_{s h}}\left(1-j \tan \phi_{Z}\right)
$$

where

$$
\tan \phi_{Z} \equiv-Q_{o} \frac{\omega^{2}-\omega_{o}^{2}}{\omega \omega_{o}} \approx-2 Q_{o} \frac{\Delta \omega}{\omega_{o}}
$$

The condition for making the total imaginary component of the impedance, as given in equation (2), zero is

$$
\tan \phi_{Z}=\left|\frac{I_{B}}{V_{C}}\right| R_{L} \cos \phi_{S}
$$

\section{TRANSMISSION LINE MATCHING}

When a mismatch occurs because the load impedance is not equal to the characteristic impedance of the transmission line, some of the wave is reflected. The reflection coefficient is defined as the ratio of the amplitudes of the forward and backward wave,

$$
\Gamma=\frac{V_{L^{-}}}{V_{L^{+}}}=\frac{Z_{L}-R_{0}}{Z_{L}+R_{0}}=\frac{\bar{Z}_{L}-1}{\bar{Z}_{L}+1} \leq 1
$$

where $\bar{Z}_{L}=\frac{Z_{L}}{Z_{0}}$, is the normalized load impedance.

Superposition of the forward and reflected waves leads to a standing wave pattern. A quantity that can actually be measured is the voltage standing wave

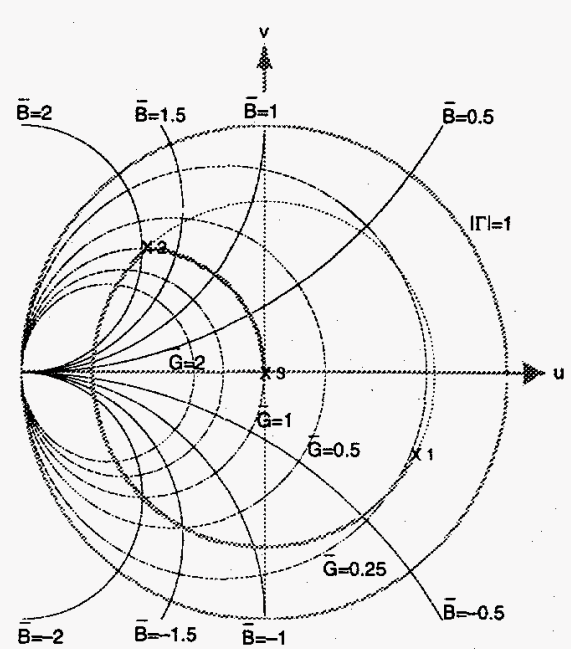

Figure 3: Smith chart representation of the reflection coefficient from a load with mismatched admittance at point 1 . A length of waveguide, $d$, transforms it on a circle to point 2 where a stub of length $d_{I}$ transforms it along a line of constant conductance to a match at 3 .

ratio (VSWR), $S$, that is related to the reflection coefficient by

$$
S=\frac{\left|V_{\max }\right|}{\left|V_{\min }\right|}=\frac{1+|\Gamma|}{1-|\Gamma|}
$$

The impedance properties of a transmission line can also be represented graphically on a Smith chart. At the load, the reflection coefficient will be designated $\Gamma_{L}$, so that at a distance $l$ from the load

$$
\Gamma(l)=\left|\Gamma_{L}\right| e^{-2 j \beta l}, \quad \beta=\frac{2 \pi}{\lambda}
$$

Equation (7) may now be rearranged to give the normalized input admittance, located at a distance $l$ from the load,

$$
\overline{Y_{I}}=\frac{1}{\overline{Z_{I}}}=\frac{1-\Gamma(l)}{1+\Gamma(l)}=\bar{G}+j \bar{B}
$$

where $\bar{G}$ is the normalized input conductance and $\bar{B}$ is the normalized input susceptance. The load admittance as defined by equation (2) can be represented as a point on the Smith chart in figure 3, for a given waveguide impedance $Z_{o}$.

Moving away from the load a distance $l$ toward the generator is equivalent to a clockwise rotation of a point on the chart through an angle $2 \beta l$ at a constant radius, allowing the transformed resistance and reactance of the load to simply read off the graph. Adding a length, $d$, of shorted line which is terminated in zero resistance has the effect of adding an admittance

$$
\overline{Y_{S}}=\frac{Y_{S}}{G_{0}}=0+\frac{j}{\tan \beta d}
$$

which is equivalent to moving along a circular contour of constant conductance on the Smith chart. In the next section we will use this graphical technique to illustrate the use of stub lines for matching a complex load impedance, like a beam loaded cavity, to a generator. Note that a matched load impedance is equivalent to a normalized resistance of 1 and zero 


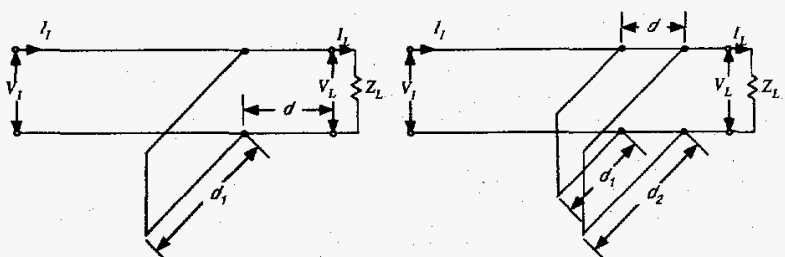

Figure 4: Single (left) and double stub matching(right). reactance, corresponding to a point at the origin of the Smith chart.

\subsection{Single stub matching}

The principle of matching with a single stub lies in choosing the length, $d_{l}$, of the stub and its distance, $d$, from the load such that the reflected wave from the stub just cancels the reflected wave from the load, as shown in figure $4 \mathrm{a}$. The procedure for choosing $d$ and $d_{I}$ was shown graphically in figure 3 . Alternatively, if the VSWR from the load is measured before the addition of the stub line the values for $d$ and $d_{l}$ can be determined from $S$,

$$
\cot \beta d_{1}= \pm \frac{S-1}{\sqrt{S}}, \quad \tan \beta d_{m}= \pm \sqrt{S}
$$

where $d_{m}$ is now the distance of the stub line measured toward the generator from the position of the voltage maximum. The positive square root sign is used for $0<d_{m}<\lambda / 4$ and is negative for $\lambda / 4<d_{m}<\lambda / 2$.

\subsection{Double stub matching}

It is usually not possible to vary the distance of the stub line from the load to compensate for load impedance changes. This difficulty can be overcome by using two adjustable stub lines of length $d_{1}$ and $d_{2}$ as the two variables, at an arbitrary distance from the load but separated by a distance $d \neq \lambda / 2$ from each other, as shown in figure $4 \mathrm{~b}$. The distance of the first stub from the load is completely arbitrary as the transformed load impedance at that location is now taken as the new load impedance to be matched.

The matching procedure is shown graphically in figure 5, where point 1 represents the normalized load admittance at the junction of the stub $d_{2}$. The stub line of length $d_{2}$ adds susceptance only, so the locus moves to point 2 along a contour of constant $\bar{G}=\bar{G}_{L}$. The length of transmission line $d$ between the two stubs transforms point 2 through an angle $2 \beta d$ where it intercepts the circle $\bar{G}=1$ at point 3 . The stub line $d_{1}$

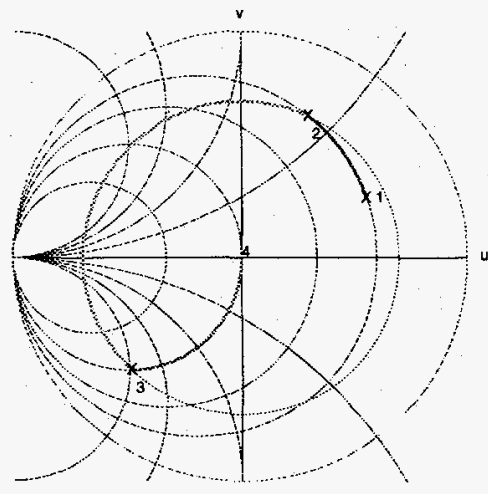

Figure 5: Smith chart representation of double stub matching.

$$
\cot \beta d_{1}= \pm \sqrt{S-1}, \quad \cot \beta d_{2}= \pm \frac{\sqrt{S-1}}{S}
$$

If the range of load impedances to be matched is unusually large, or if it is physically difficult to make $d$ small enough to accommodate the range of impedances, then a third adjustable stub can be added. Adding a third degree of freedom in a triple stub matching arrangement is sufficient to ensure all load conditions can be matched.

\section{BEAM STABILITY}

At first sight it might appear that not detuning the cavity would be advantageous for the driving terms of the coupled bunch instability. However, the beam sees both the cavity and generator impedance in parallel and the generator now appears as a reactive impedance when transformed through the stub line. In fact, the beam must see exactly the same reactive impedance component as the conventionally detuned cavity. The main advantage remains the practical aspect of placing the moveable tuning element outside of the vacuum system in the waveguide.

(a)
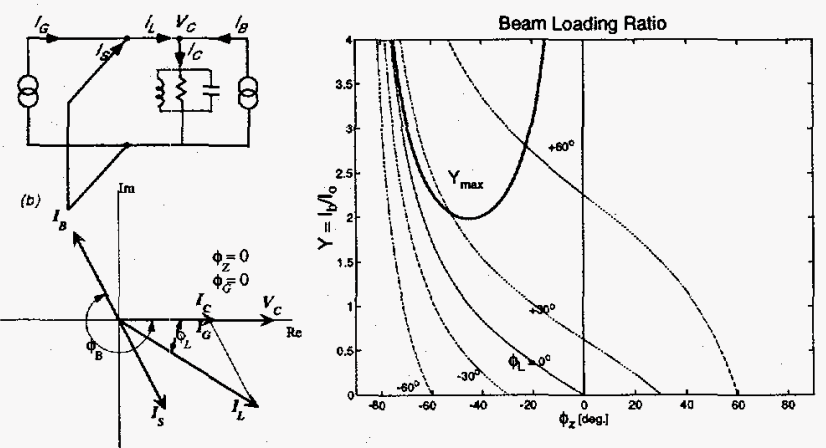

Figure 6: Equivalent circuit (a) with stub line matching and the phasors (b) including the stub line current. Beam loading limit (right) as a function of impedance angle and loading angle.

Similarly, the Robinson beam loading limit, according to

$$
Y_{\max }=\frac{2 \sin \phi_{B}}{\sin 2 \phi_{Z}}, \quad \tan \phi_{L}=\frac{-\tan \phi_{Z}+Y \cos \phi_{B}}{1-Y \sin \phi_{B}}
$$

appears to increase when $\phi_{z}=0$, as in fig. 6. However, stability is now lost when $V_{L}$ is $180^{\circ}$ out of phase with $I_{B}$, even though $V_{G}$ is in phase with $V_{C}$, so the beam loading limit is the same as for conventional matching.

\section{REFERENCES} to move the point along the contour $\bar{G}=1$ until it meets the origin at point 4.

As with the single stub, the matching conditions can be expressed in terms of the measured VSWR, so that in the example for $d=\lambda / 4$ :

\section{F. Pedersen, "RF Cavity Feedback", Proceedings of} B Factories, SLAC-400, pp. 192-207.

2. K. W. Robinson, CEA Report CEAL-1010, 1964.

3. F. Pedersen, IEEE Trans. on Nucl. Sci. Vol NS-32, 1985 pp. 2138-40.

4. P. B. Wilson, AIP Conf. Proc. $\underline{87}$, p.450-563; SLACPUB-2884 1982.

5. R. E. Collin, "Foundations of Microwave Engineering", 2nd ed. 1992, McGraw Hill

6. A. Gamp, Proc. 1991 CERN Accel. School, CERN 92-03, p. 396-415.

7. E. Raka, AIP Conf. Proc. $\underline{184}$, p.288-342 


\section{DISCLAMMER}

Portions of this document may be illegible in electronic image products. Images are produced from the best available original document. 